\title{
Variable Structure Control of a Hand-Launched Glider
}

\author{
Mark R. Anderson* \\ Paper Pilot Research, Inc., Sterling, VA 20165-0776 USA \\ Martin R. Waszak ${ }^{\dagger}$ \\ NASA Langley Research Center, Hampton, VA 23681-2199 USA
}

Variable structure control system design methods are applied to the problem of aircraft spin recovery. A variable structure control law typically has two phases of operation. The reaching mode phase uses a nonlinear relay control strategy to drive the system trajectory to a pre-defined switching surface within the motion state space. The sliding mode phase involves motion along the surface as the system moves toward an equilibrium or critical point. Analysis results presented in this paper reveal that the conventional method for spin recovery can be interpreted as a variable structure controller with a switching surface defined at zero yaw rate. Application of Lyapunov stability methods show that deflecting the ailerons in the direction of the spin helps to insure that this switching surface is stable. Flight test results, obtained using an instrumented hand-launched glider, are used to verify stability of the reaching mode dynamics.

\section{Nomenclature}

$\begin{array}{lll}b & =\text { wing span } \\ c_{b a r} & =\text { mean aerodynamic chord } \\ h & =\text { angular momentum vector } \\ I_{x} & =\text { roll moment of inertia } \\ I_{y} & =\text { pitch moment of inertia } \\ I_{z} & =\text { yaw moment of inertia } \\ k & =\text { relay gain } \\ L & =\text { total rolling moment } \\ L_{A} & =\text { aerodynamic rolling moment } \\ L_{\delta A} & =\text { rolling moment due to aileron deflection } \\ M & =\text { total pitching moment } \\ M_{A} & =\text { aerodynamic pitching moment } \\ M_{\delta E} & =\text { pitching moment due to elevator deflection } \\ m_{x} & =\text { longitudinal magnetic field strength } \\ m_{y} & =\text { lateral magnetic field strength } \\ m_{z} & =\text { vertical magnetic field strength } \\ N & =\text { total yawing moment } \\ N_{A} & =\text { aerodynamic yawing moment } \\ N_{\delta R} & =\text { yawing moment due to rudder deflection } \\ p & =\text { roll rate } \\ q & =\text { pitch rate } \\ q_{b a r} & =\text { dynamic pressure } \\ r & =\text { yaw rate } \\ R & =\text { servo rate limit } \\ S & =\text { reference area }\end{array}$

\footnotetext{
* Technical Director, P.O. Box 650776, AIAA Senior Member.

${ }^{\dagger}$ Senior Research Engineer, Dynamic Systems and Control Branch, MS 132, AIAA Associate Fellow.
} 


$\begin{array}{ll}t & =\text { time } \\ u & =\text { control vector } \\ V & =\text { Lyapunov function } \\ x & =\text { state vector } \\ y & =\text { subspace vector } \\ \Delta & =\text { control law update rate } \\ \delta_{A} & =\text { aileron deflection } \\ \delta_{E} & =\text { elevator deflection } \\ \delta_{R} & =\text { rudder deflection } \\ \varepsilon & =\text { servo deadband } \\ \sigma & =\text { switching surface } \\ v & =\text { control deflection noise }\end{array}$

\section{Introduction}

$\mathrm{I}_{\mathrm{a}}$

T has long been known that the most effective spin recovery technique for fuselage-heavy aircraft is to use the Iailerons to roll the aircraft into the spin. This technique was first proven effective by A.I. Neihouse in 1940, using scale models in the free-spinning wind tunnel at Langley Aeronautical Laboratory. ${ }^{1}$ This recovery strategy might be considered counterintuitive because the pro-spin aileron deflection will tend to aggravate the roll rate component of the aircraft's angular momentum. The objective of this paper is to explore this well-known spin recovery strategy using a recently-developed integrated toolset for modeling and analysis of highly dynamic aircraft motions

The integrated toolset combines existing nonlinear methods for aerodynamic modeling, stability analysis, and control system design. The nonlinear lifting line aerodynamic modeling method was first introduced by Piszkin and Levinsky in 1975. ${ }^{2}$ This method differs from conventional lifting-line methods in that it allows the section lift coefficient to be expressed as a nonlinear function of angle-of-attack. This extension yields accurate aerodynamic information over a wide angle-of-attack range.

Hsu introduced the cell-to-cell mapping method for nonlinear system stability analysis in $1987 .^{3}$ The power of the cell-to-cell mapping theory comes from the fact that the map itself represents a linear Markov chain. Consequently, linear system stability analysis tools can be applied to the map and the results reveal how the underlying nonlinear system will behave.

Variable Structure Control (VSC) is a high-speed switching feedback control technique that has been developed for control of nonlinear dynamic systems. ${ }^{4}$ This method was added to the integrated toolset because the relay control behavior of VSC is very similar to automatic upset recovery systems used on full-scale aircraft. For example, some fighter aircraft are equipped with a spin arrow display that requires the pilot to apply full control authority in the direction of the spin arrow.

The nonlinear lifting line aerodynamic modeling method and the subspace cell-to-cell mapping method are described in greater detail in a recent contractor report. ${ }^{5}$ The contractor report also provides a user guide to the software that has been developed for each method. These software programs were used to generate the results described in this paper.

A brief review of the subspace cell-to-cell mapping method is provided in Section II of this paper. Section III describes how coventional spin recovery control strategies are related to variable structure control laws. Section IV describes the hand-launched glider that was used to gather flight test data. Section V presents analysis and flighttest results. Several important conclusions are offered in Section VI.

\section{Subspace Cell-to-Cell Mapping}

The cell-to-cell map represents the transition probability of solution trajectories moving from one cell to another. The method works well for systems of dynamic order less than about five. When the dynamic order exceeds five, the number of cells needed to accurately represent the system dynamics becomes prohibitively large. The approach used in this research has been to define a two-dimensional subspace within the state-space domain of interest. The cell-to-cell mapping method is then used to create a cell map on the two-dimensional subspace.

The subspace mapping approach is illustrated in Fig. 1 for a third-order system. The state variables are $x_{1}, x_{2}$, and $x_{3}$. The two-dimensional subspace is defined by the variables $y_{1}$ and $y_{2}$, such that $x_{1}=y_{1}, x_{2}=y_{2}$, and $x_{3}=0$. The two-dimensional subspace is divided into twenty-four cells in Fig. 1. The cell-to-cell map for this system is constructed by starting several trajectories from within each cell. These trajectories are propagated forward for a fixed time period. The point at the end of the trajectory is projected onto the two-dimensional subspace and the cell in which this point lands is recorded. As Fig. 1 illustrates, solutions that start from the one cell do not necessarily 
land in the same cell. These differences are captured in the transition probabilities that form the cell-to-cell map. For example, if 100 trajectories are started from cell \#2, and 40 of those ends in cell \#4, then the $(4,2)$ element of the cell-to-cell map is set to $40 / 100$

The cell-to-cell map that is created on the two-dimensional subspace is obviously not sufficient to capture the stability properties of the entire state-space. However, significant insight can be gained by locating and orienting the subspace near features of interest. One example is to study the behavior of the system near a critical point by defining several orthogonal subspaces that

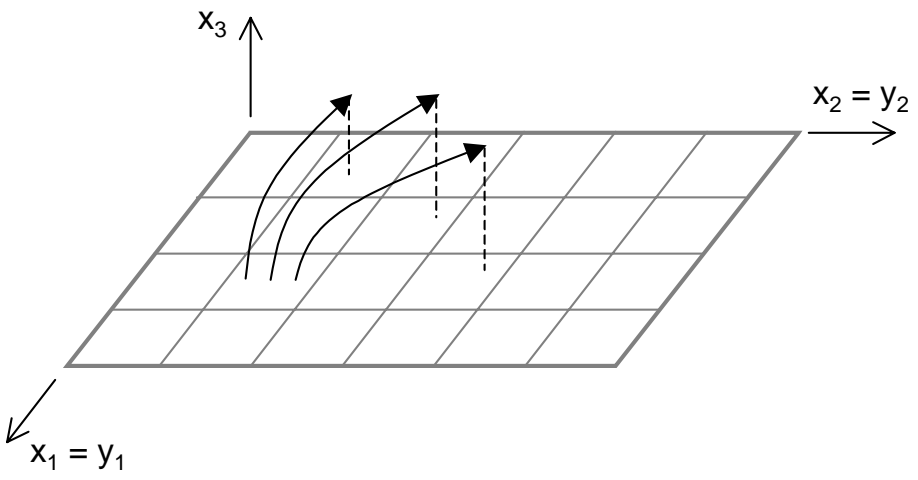

Figure 1. Subspace Cell-to-Cell Map Construction intersect the critical point.

In analyzing cell-to-cell map properties, Hsu devised ways to sort and categorize how solution trajectories enter and leave the cells. Two of the categories that are most relevant to this research are absorbing cells and transient cells. A transient cell has the property that once a solution leaves the cell, it does not return. An absorbing cell has the property that once a solution enters the cell, it does not leave. Stable critical points in the nonlinear system statespace are typically found near one or more absorbing cells.

It is possible to locate the absorbing cells by studying the long-term behavior of the Markov chain. The list of transient cells that have a high probability of moving into an absorbing cell determine the basin of attraction for the absorbing cell. Solutions that start in transient cells located near the edge of an attraction basin would presumably take a longer time to reach the absorbing cells. The expected absorption time is used to quantify this relationship. Absorbing and transient cells will be identified in subsequent analysis results using plots of the expected absorption time from within the two-dimensional subspace.

\section{Variable Structure Spin Control}

The VSC control strategy is divided into two phases. The first phase of the control strategy is designed to drive the state trajectory onto a specified subspace called the switching surface. Behavior of the closed-loop system during this phase is often called the reaching mode. The second phase of the VSC control strategy defines the system behavior while the system is operating on or near the switching surface. This phase is called the sliding mode. A linear state feedback structure is generally used for sliding mode control. Its objective is to drive the system state to a desired equilibrium point by "sliding" the trajectory along the switching surface.

This paper will focus on the reaching mode phase of the VSC control technique. A constant gain relay is one of the most common elements used to implement reaching mode control laws. Assume that the function $\sigma(x)=0$ represents a switching surface. If $x$ is the state vector and $u$ is the control variable, the constant gain relay control law is given in Eqs. (1) and (2).

$$
\begin{gathered}
u=k \operatorname{sgn}[\sigma(x)] \\
\operatorname{sgn}[\sigma(x)]= \begin{cases}+1, & \sigma(x)>0 \\
-1, & \sigma(x)<0\end{cases}
\end{gathered}
$$

The design philosophy of the VSC reaching mode is to insure that the domain of attraction to the switching surface is as large as possible. This objective is achieved by choosing switching surfaces such that asymptotic stability can be guaranteed using the Lyapunov's direct method. A Lyapunov function $V(x)$ is defined in terms of the switching surface, as shown in Eq. (3). The Lyapunov function $V(x)$ is positive definite for all nonzero $x$ and $V(0)=$ 
0 is satisfied as long as $\sigma(0)=0$. Under these conditions, the system state trajectory will converge to the switching surface as long as the control law is designed to keep $d V / d t<0$. The time derivative of $V(x)$ yields the global stability condition relative to the switching surface.

The reaching mode Lyapunov stability condition, expressed in Eq. (4), will be used in this paper to investigate control strategies for aircraft spin recovery. The objective of the VSC control law will be to drive the aircraft away from the spin critical point and toward a stable switching surface. For aircraft spin control, it would seem logical to design switching surfaces that are related to the aircraft's angular momentum. Relative to a conventional body-fixed axis system, the aircraft's angular momentum vector is written as shown in Eq. (5). ${ }^{6}$ Note that it has been assumed that the cross-products of inertia are small.

Separate switching surfaces are defined for each component of the angular momentum vector in Eqs. (6) - (8). The associated Lyapunov stability condition for each switching surface is listed in Eqs. (9) - (11). Note that crossaxis control effects, such as yawing moment due to aileron deflection, have been ignored for simplicity.

$$
\begin{aligned}
& V(x)=\sigma^{2}(x) / 2 \\
& d V / d t=\sigma d \sigma / d t<0 \\
& h=\left[\begin{array}{lll}
I_{x} p & I_{y} q & I_{z} r
\end{array}\right]^{T} \\
& \sigma_{1}=I_{x} p \\
& \sigma_{2}=I_{y} q \\
& d V_{1} / d t=\sigma_{1} d \sigma_{1} / d t=I_{x} p\left[\left(I_{y}-I_{z}\right) q r+q_{b a r} S b\left(L_{A}+L_{\delta A} \delta_{A}\right)\right] \\
& d V_{2} / d t=\sigma_{2} d \sigma_{2} / d t=I_{y} q\left[\left(I_{z}-I_{x}\right) p r+q_{b a r} S c_{b a r}\left(M_{A}+M_{\delta E} \delta_{E}\right)\right] \\
& d V_{3} / d t=\sigma_{3} d \sigma_{3} / d t=I_{z} r\left[\left(I_{x}-I_{y}\right) p q+q_{b a r} S b\left(N_{A}+N_{\delta R} \delta_{R}\right)\right]
\end{aligned}
$$

Spin recovery strategies can be developed by considering the effect of constant gain relay control laws on the stability conditions defined above. First consider Eq. (12), which is a constant gain relay control law for the rudder. The gain $k_{3}$ is set equal to the maximum rudder deflection $\delta_{\text {Rmax }}$. This control strategy defines a switching surface $\sigma_{3}$ $=0$ and is governed by the Lyapunov function $V_{3}(x)$. In a right spin, for example, the yaw rate is positive. Therefore, $\sigma_{3}$ is positive in a right spin and the rudder control law will set $\delta_{R}=+\delta_{R \max }$. A negative yawing moment 
results because the rudder sign convention is such that $N_{\delta R}$ is negative. This control moment helps to force $d V 3 / d t$ negative. If $d V_{3} / d t$ remains negative, the spinning aircraft will be driven to the $\sigma_{3}=0$ switching surface, which has the net result of driving yaw rate to zero. This rudder relay control law mimics the conventional wisdom that the rudder should be deflected to oppose the spin.

$$
\delta_{R}=k_{3} \operatorname{sgn}\left[\sigma_{3}(x)\right]
$$

Now consider the constant gain control law for the aileron defined in Eq. (13). This aileron relay control law defines a switching surface at $\sigma_{1}=0$. The aileron relay control gain will first be chosen such that $k_{1}=-\delta_{\operatorname{Amax}}$. A sign convention of $L_{\delta A}>0$ implies that this aileron relay control law will deflect the aileron to generate a rolling moment in a direction opposing the spin. For example, both the roll rate $p$ and yaw rate $r$ will be positive in a right spin. The aileron relay control law will therefore command $\delta_{\mathrm{A}}=-\delta_{\text {Amax }}$ in a right spin. This command deflection will produce a negative rolling moment, which will help to force $d V_{1} / d t$ negative.

$$
\delta_{\mathrm{A}}=k_{1} \operatorname{sgn}\left[\sigma_{1}(x)\right]
$$

It would seem logical that a combination of the rudder and aileron relay control laws described above would yield an even more effective spin recovery technique. The rudder control law will tend to drive the airplane to the $\sigma_{3}=0$ surface and the aileron control law will tend to drive the airplane to the $\sigma_{1}=0$ surface. Experimental results, shown later in Section IV, reveal that these two objectives conflict, causing the airplane to oscillate between the surfaces rather than actually reach either one. The aircraft eventually reaches the intersection of the surfaces, where $\sigma_{1}=\sigma_{3}=0$, but the technique does not lead to the most rapid recovery.

The aileron will be deflected into the spin if the relay control law gain is set such that $k_{1}=\delta_{\text {Amax }}$. In a right spin, with roll rate positive, the aileron relay control law will set $\delta_{A}=\delta_{\text {Amax }}$. Because $L_{\delta A}$ is positive, this aileron setting will produce a positive rolling moment. A positive rolling moment will introduce a positive contribution to $d V_{1} / d t$. This choice in aileron control gain will tend to force the aircraft away from the $\sigma_{1}=0$ switching surface.

The value in driving the aircraft away from the $\sigma_{1}=0$ switching surface is seen by careful consideration of the inertia coupling terms in the Lyapunov stability conditions. The inertial coupling term in the $d V_{3} / d t$ stability condition is $\left(I_{x}-I_{y}\right)$ pqr. For a fuselage-heavy airplane, $I_{y}$ is larger than $I_{x}$, which means that the inertial coupling term will be negative, in a right spin, as long as the pitch rate $q$ is positive. Therefore, a beneficial effect is achieved with regard to the stability of the $\sigma_{3}=0$ switching surface if $q$ is forced positive in a right spin. Ignoring the aerodynamic pitching moment and assuming neutral elevator, the pitch acceleration equation is given by Eq. (14). Forcing the aircraft away from the $\sigma_{1}=0$ switching surface tends to increase the aircraft roll rate. Equation (14) shows that the increased roll rate will introduce pitch acceleration in the same direction. Consequently, the pitch rate will become positive in a right spin when the ailerons are deflected into the spin.

$$
I_{y} d q / d t=\left(I_{z}-I_{\chi}\right) p r
$$

The spin recovery technique discovered by Neihouse is therefore identical to a VSC strategy that centers on the $\sigma_{3}=0$ switching surface. The rudder and aileron are both used to insure stable reaching mode dynamics relative to this surface. The rudder relay control law provides a direct negative contribution to the $d V_{3} / d t$ stability condition. The pro-spin aileron relay control law provides an additional negative contribution indirectly, through inertial coupling. As a result, the airplane is expected to rapidly reach the $\sigma_{3}=0$ switching surface. After the stable switching surface is reached, the sliding mode phase of the VSC strategy would be used to complete the spin recovery. 


\section{Vehicle Description and Modeling}

A small hand-launched glider was constructed to provide test data and verify the theoretical results. The glider, shown in Fig. 2, was built by modifying a powered, remote control, aerobatic model airplane kit. The main structure of the glider is made from expanded-polypropylene foam reinforced with carbon-fiber wire. A battery tray containing four AAA batteries is mounted in the slot originally intended for the remote-control receiver batteries. The center-of-gravity for the flight-ready glider is located $20 \mathrm{~cm}$ behind the nose of the aircraft and is close to the fuselage centerline. The moments of inertia for the glider were estimated by measuring the weight and physical dimensions of each component before final construction. The heaviest components are the fuselage (74 g), battery tray (56 g), and the printed circuit board (32 g). The estimated mass and inertia characteristics are listed in Table 1.

A single experimenter can perform all of the steps necessary to flight test the glider. Setscrews on each servo control arm are used to center each control surface. Once power is connected, a green LED illuminates to serve as a power indicator. A handheld computer can be attached to the printed circuit board using a four-pin modular connector. A software program written for the handheld computer allows the experimenter to download and store data from the airplane. It can also be used to change the control sequence to be flown

\section{Table 1. Glider Geometry and Mass Properties}

Wing Span, b................................................. $76.5 \mathrm{~cm}$ Length ........................................................... $65.0 \mathrm{~cm}$

Wing Reference Area, S.............................. $1400 \mathrm{~cm}^{2}$

Mean Aerodynamic Chord, $\mathrm{C}_{\mathrm{bar}}$ ……………..... $18.5 \mathrm{~cm}$

Aspect Ratio .............................................................. 4.2

Mass ........................................................ $0.226 \mathrm{~kg}$

Roll Moment of Inertia, $\mathrm{I}_{\mathrm{x}} \ldots \ldots \ldots \ldots \ldots \ldots \ldots . . . . . . .16 .2 \mathrm{~kg}-\mathrm{cm}^{2}$

Pitch Moment of Inertia, $\mathrm{I}_{\mathrm{y}} \ldots \ldots \ldots \ldots \ldots \ldots \ldots . . . . . . . . .11 .8 \mathrm{~kg}-\mathrm{cm}^{2}$

Yaw Moment of Inertia, $\mathrm{I}_{\mathrm{z}} \ldots \ldots \ldots \ldots \ldots \ldots \ldots . . . . . . . . . . .45 .4 \mathrm{~kg}-\mathrm{cm}^{2}$ during the next test.

A flight test begins when the experimenter presses a momentary reset switch located on the circuit board. A yellow LED illuminates to indicate that the circuit board is now recording data. A pulse input is applied to the left aileron after a two-second delay. This aileron pulse signals the experimenter to launch the glider. The on-board microcontroller will begin execution of the programmed control sequence two seconds after the aileron pulse. Each flight lasts from five to eight seconds, depending on the control sequence, initial velocity, and initial altitude. The yellow LED is automatically turned off, and data is no longer collected, after a time period of fifteen seconds. Subsequent test flights can be initiated by pressing the reset switch again. Data collected from each flight is appended to the data from previous flights. More than sixty flights can be stored before the handheld computer is needed to download the data and to reset the flash memory.

The glider is equipped with an application-specific instrumentation board. The printed circuit board was developed to collect data and control the glider in flight. The circuit is controlled by a microcontroller that is driven by a $20 \mathrm{MHz}$ crystal and includes a multi-channel 10-bit analog-todigital (A/D) converter. The microcontroller drives up to four servos. For this application, micro-miniature servos are used to control the left aileron, right aileron, rudder, and elevator.

A yaw rate gyro is mounted near the center of the circuit board. This gyro has a dynamic range of $\pm 300 \mathrm{deg} / \mathrm{s}$. Using the 10 bit A/D converter of the microcontroller results in a yaw rate resolution of better than $1 \mathrm{deg} / \mathrm{s}$. Magnetic field strength measurements are obtained using three

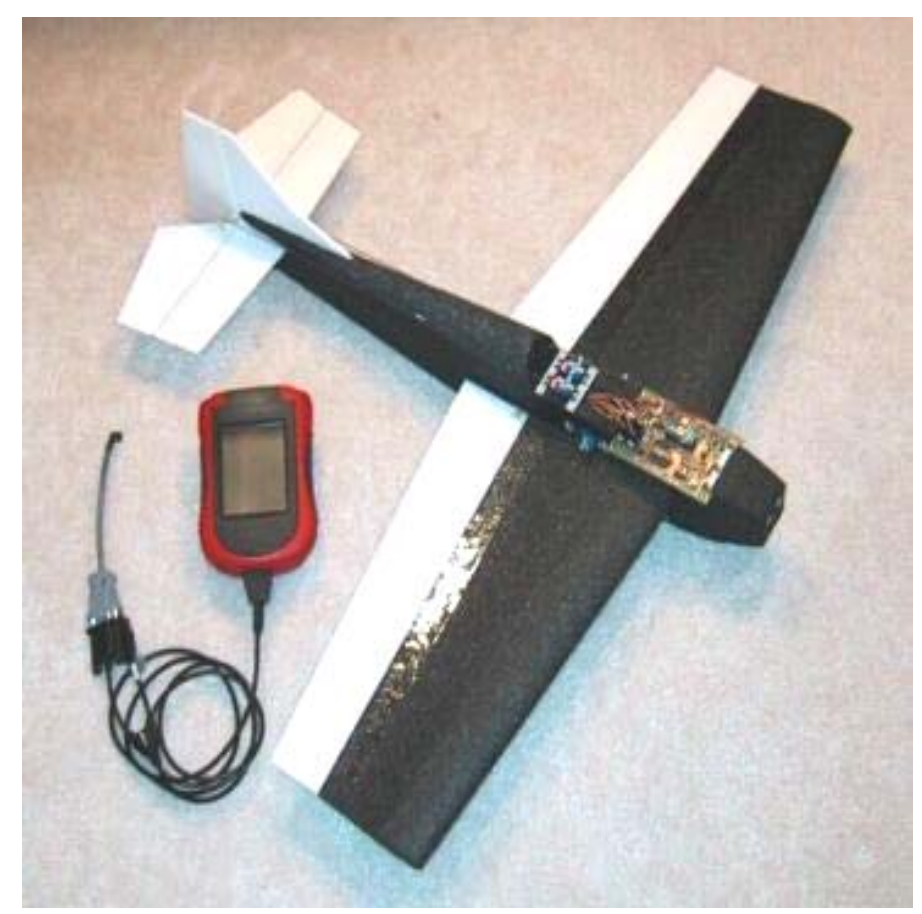

Figure 2. Hand-Launched Glider 
magnetoresistive sensors. Single-supply instrumentation amplifiers amplify the differential voltage output from these sensors. This combination leads to a minimum resolution of about 150 nano-Teslas. The combination of three magnetoresistive sensors forms a three-axis magnetometer. The magnetometer was calibrated by comparing its response to the IGRF2000 geodetic model published by the National Geographic Service.

The VSC spin recovery control laws require estimates of $\operatorname{sgn}\left[\sigma_{1}(x)\right]$ and $\operatorname{sgn}\left[\sigma_{3}(x)\right]$. The sign of the yaw rate gyro signal is taken as the value of $\operatorname{sgn}\left[\sigma_{3}(x)\right]$. The value of $\operatorname{sgn}\left[\sigma_{1}(x)\right]$ is estimated using the magnetoresistive sensors. The rate of change in the lateral magnetoresistive signal is related to the body-axis angular rates through Eq. (15). Because $r$ is already measured by the yaw rate gyro, Eq. (15) can be solved for roll rate $p$. The sign of the roll rate estimate in Eq. (16) is assigned to $\operatorname{sgn}\left[\sigma_{1}(x)\right]$. A backward difference approximation was used to estimate the magnetometer signal rate $d m_{y} / d t$. The backward difference expression shown in Eq. (17) where $m_{y}(k)$ is the current lateral magnetic sensor reading, $m_{y}(k-1)$ is the previous sensor reading, and $\Delta$ is the time difference between readings.

$$
\begin{gathered}
d m_{y} / d t=-r m_{x}+p m_{z} \\
\operatorname{sgn}[p]=\operatorname{sgn}\left[d m_{y} / d t+r m_{x}\right] \operatorname{sgn}\left[m_{z}\right] \\
d m_{y}(k) / d t \cong\left[m_{y}(k)-m_{y}(k-1)\right] / \Delta
\end{gathered}
$$

Sensor data are stored on a flash memory chip. The nonvolatile flash memory chip is organized with 2048 pages of 264 bytes each ( $540 \mathrm{~KB}$ total). Each data record contains twelve bytes and data records are written at a rate of 40 records per second. As a result, sensor and control data are written to the flash memory chip at a rate of 480 bytes per second. The forward difference approximation in Eq. (17) is updated at a rate of only 4 times per second ( $\Delta=0.25 \mathrm{~s}$ ). The VSC control laws are updated at a rate of 4 times per second as well.

The dynamic model for the control servos is shown in Fig. 3. During bench testing, it was noted that the servos responded to maximum amplitude position commands by moving at a nearly constant rate. The constant control rate $R=2.8 \mathrm{rad} / \mathrm{s}(160 \mathrm{deg} / \mathrm{s})$ was set by observing that each control surface travels forty degrees in $0.25 \mathrm{~s}$. A deadband of $\varepsilon=0.03 \mathrm{rad}$ ( $2 \mathrm{deg}$ ) is also assumed. A random noise signal is added to each control surface position for postflight data processing. This zero-mean, Gaussian noise signal $v$ is intended to model nonlinearity in the control surface linkage caused by control-rod flexing, friction, and control horn backlash. The intensity of the control surface noise is set to $0.01 \mathrm{~s}^{-1}$.

A nonlinear filtering method is used to reconstruct motion state variables that are not measured in flight. Extended Kalman Filters (EKF) have been traditionally used for this purpose. The EKF linearizes the aircraft motion model and measurement functions, relative to the current mean state, so that a linear Kalman filter can be utilized. Its obvious limitations are that the motion dynamics must be predominately linear and

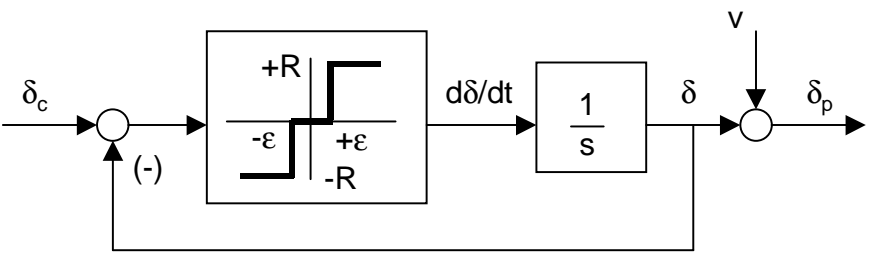

Figure 3. Servo Model

the noise sources must be Gaussian. These limitations make the EKF less suitable for reconstructing state trajectories from highly dynamic maneuvers. To improve performance, the Unscented Kalman filter (UKF) was adopted for this application. The UKF utilizes a set of special vectors, called sigma-points, to represent the state probability distribution. ${ }^{7}$ The UKF is very similar to the square-root implementation of the EKF because the sigmapoints are constructed using the square root of the state covariance matrix. The computational burden of the UKF is therefore similar to the EKF, which makes it an ideal substitute for the EKF. Standard equations of motion are used to represent the glider dynamics and quaternions are used to describe the aircraft's attitude. 


\section{Analysis and Flight Test Results}

The nonlinear lifting line software was used to estimate a complete aerodynamic model for the hand-launched glider. The wing was divided into two distinct aerodynamic surfaces and the vortex structure for each wing surface is solved separately. Each wing surface is divided into seven sections and five rows of wake vortices are used for each surface. The horizontal tail is also split into two separate surfaces, with four sections for each surface. The horizontal tail surfaces are affected by the wake of the wing surfaces. The vertical tail has four sections as well. The wing airfoil section is the NACA 0012. ${ }^{8}$ The tail surfaces are modeled using the NACA 0006 airfoil section.

The estimated lift coefficient characteristics predict that stall occurs at approximately twenty-four degrees angleof-attack. The static margin is about 0.12 . Conventional flight dynamic modes are readily identified using spectral analysis of the glider model linearized about an equilibrium glide condition. Figure 4 shows the glider characteristic roots as a function of angle-of-attack for a constant airspeed of $6 \mathrm{~m} / \mathrm{s}$. Each dot represents a two-degree increment in the range from six to thirty degrees angle-of-attack.

For smaller angles-of-attack, the roll mode time constant is about $0.05 \mathrm{~s}$ and the spiral mode time constant is $50.0 \mathrm{~s}$. The Dutch-roll mode has a natural frequency of $4.6 \mathrm{rad} / \mathrm{s}$ and a damping ratio of 0.6. The phugoid mode is stable, and has a natural frequency of $1.4 \mathrm{rad} / \mathrm{s}$ with a damping ratio of 0.4. Two stable real roots, located at -2.2 and $14.2 \mathrm{rad} / \mathrm{s}$, define the short-period mode.

The aircraft remains stable as the angle-ofattack is increased beyond stall. The characteristic roots associated with the two longitudinal modes move toward one another. The natural frequency of the phugoid mode increases to about $2.5 \mathrm{rad} / \mathrm{s}$ while the short-period real roots merge to form a set of complex-conjugate roots with natural frequency of $9.1 \mathrm{rad} / \mathrm{s}$ and damping ratio 0.45 . The Dutch roll mode roots move slightly closer to the origin. The time constant of the roll mode is increased and the time constant of the spiral mode root is decreased.

Data from over one hundred test flights of the hand-launched glider was collected. The test flights were conducted over a period of several weeks, in calm winds, and at temperatures above

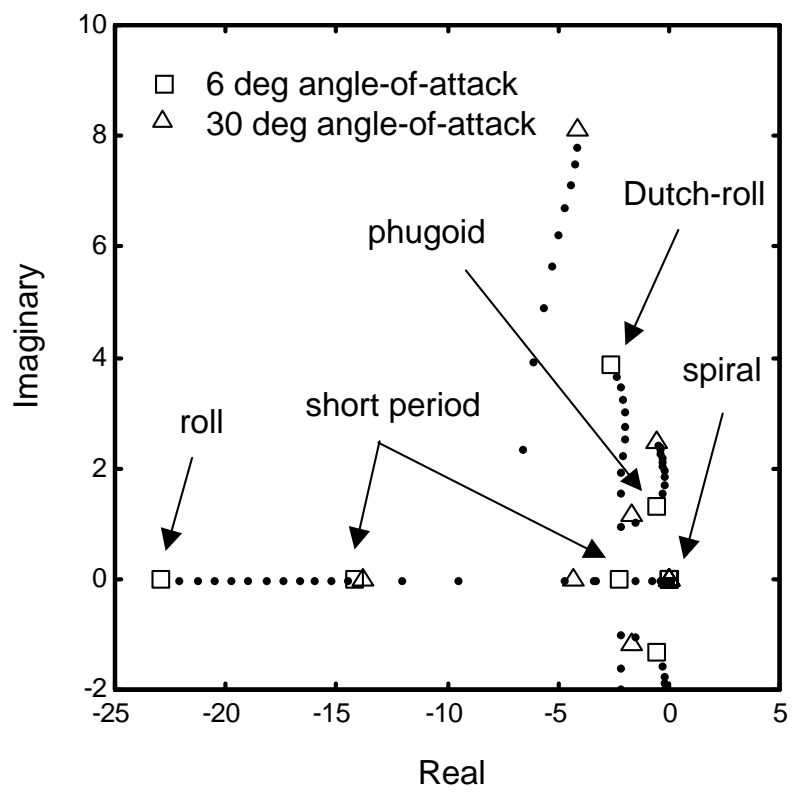

Figure 4. Glider Characteristic Roots $70^{\circ} \mathrm{F}$. Standard sea level conditions were assumed for all data processing because the test field elevation was approximately $60 \mathrm{~m}$ above sea level. Data collected during each flight was post-processed using the UKF method described previously. Solutions were obtained for approximately one-half of the test flights. The majority of the unsuccessful cases resulted when the state covariance matrix of the UKF became negative definite. This problem generally occurred at very large sideslip angles and might be caused by inadequate aerodynamic modeling of the glider fuselage.

Flight testing was conducted in two phases. The first phase involved a series of functional test flights to verify sensor calibration and control surface movement. These tests were also used to compare the recorded sensor readings to predictions from the aerodynamic model. The control deflection inputs for the functional test flights included aileron doublets, rudder doublets, and elevator pulses. The second phase of flight testing was devoted to the evaluation of the VSC reaching mode control laws.

Figure 5 shows an example functional test flight result. The glider response to an aileron doublet input is depicted in this figure. The solid line is the UKF prediction while the dots represent the sensor measurements. The dashed line is the aileron command provided by the on-board microcontroller. The aileron doublet is applied four seconds into the flight. The aircraft immediately rolls to a nearly inverted attitude as a result of the doublet input. The vertical magnetic and yaw rate sensor readings are shown as dots in the lower half of Fig. 5. These measurements are reasonably close to the response predicted by the aerodynamic model and the UKF. Angular rates on the order of $100 \mathrm{deg} / \mathrm{s}$ are measured by the yaw rate gyro and predicted by the model. The response of the vertically mounted magnetic sensor is also shown to be comparable with model predictions. 

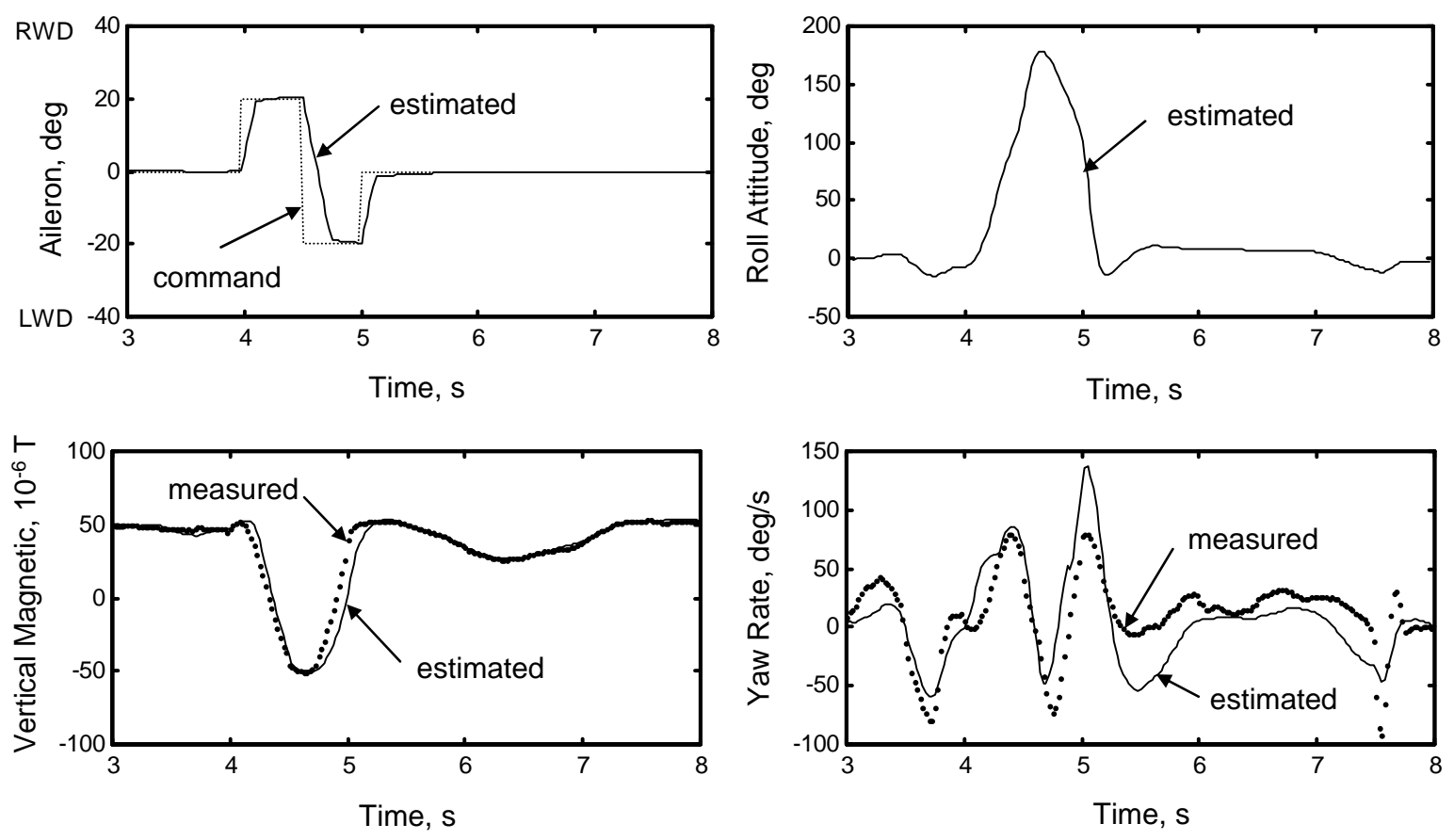

Figure 5. Aileron Doublet Response

The subspace cell-to-cell mapping analysis of the spin recovery control strategies will focus on a twodimensional subspace that is aligned with yaw rate and roll rate. The absorption times for cells in this subspace are shown in Fig. 6a for neutral control settings and Fig. 6b for spin entry controls. The transient cells are shaded in Figure 6 such that a darker shade of blue represents a longer absorption time. Two absorbing cells are found near the origin of Fig. 6a. They are left unshaded in Fig. 6a. These absorbing cells are associated with the constant speed equilibrium glide condition. The spin entry assumes control settings for a right spin: elevator 20 degrees trailing edge up, ailerons 20 degrees right wing down, and rudder 20 degrees trailing edge right. A single absorbing cell is found in Fig. 6b. This absorbing cell locates the predicted conditions for a steady right spin.

The short flight duration and low initial altitude prevented the aircraft from settling in a clearly defined steady spin condition. However, flight test results confirm that the glider was able to reach the critical spin conditions predicted by the cell maps. Two spin entry trajectories, obtained from glider flight testing, are shown in Fig. 7. The open circles in these plots mark where spin entry controls are applied. The open squares denote where the spin recovery control laws are initiated. Both trajectories are clearly attracted to the location of the steady spin absorbing cells. The path of the two trajectories appears, at first glance, to be vastly different. However, closer inspection reveals very similar characteristics in both trajectories. The differences in path are likely due to slightly different initial conditions when the spin entry controls are first applied. 


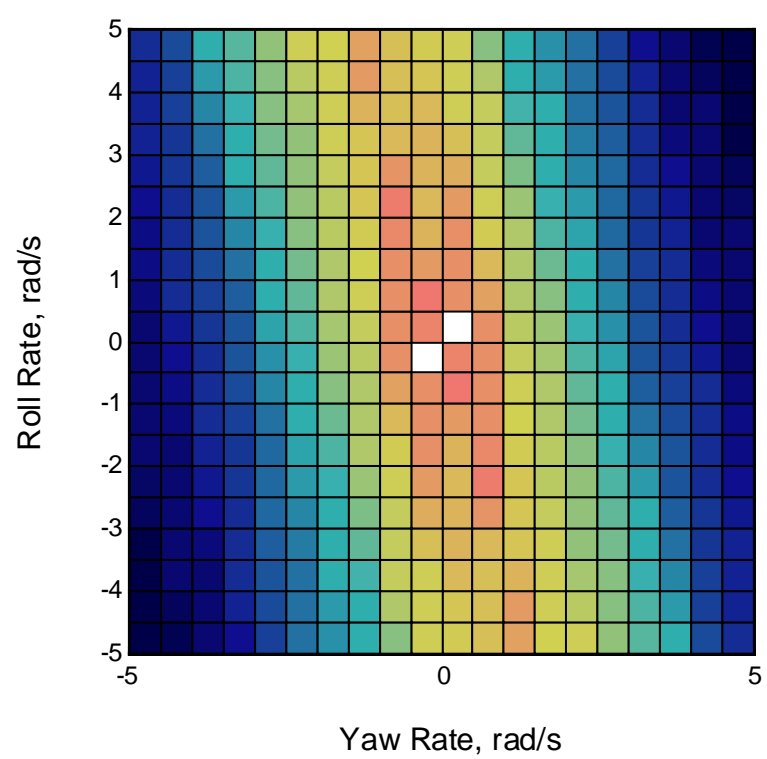

a) Neutral Controls

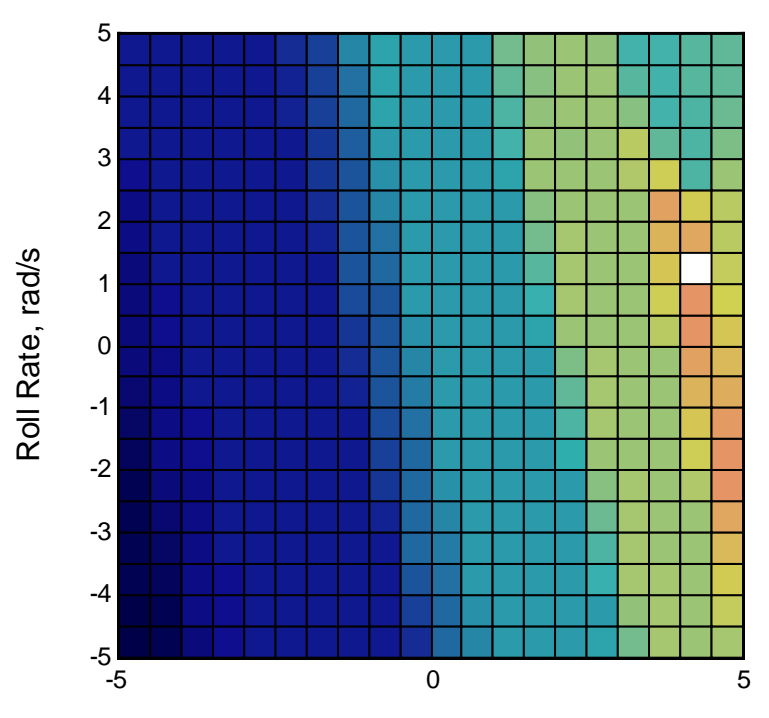

Yaw Rate, rad/s

b) Spin Entry Controls

Figure 6. Cell Maps for Neutral and Spin Entry Controls
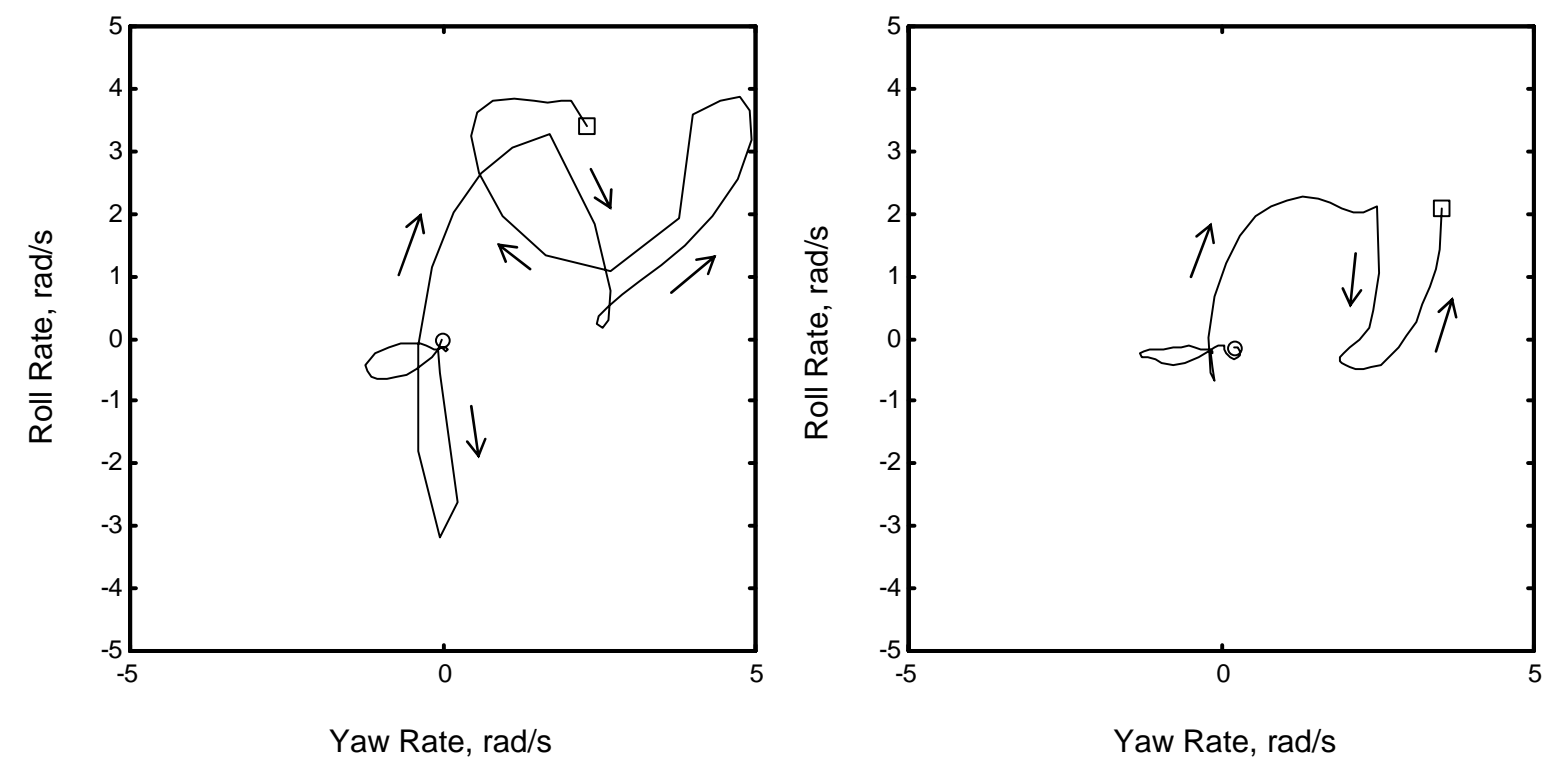

Figure 7. Spin Entry Trajectories from Flight Test

Two VSC recovery control laws are evaluated in the next series of figures. The first recovery strategy uses a constant gain relay rudder control to oppose the spin and a constant gain relay aileron control, also deflected to oppose the spin. This strategy, denoted RAA (rudder+aileron against), defines control deflections in the direction of the switching surfaces $\sigma_{1}=0$ and $\sigma_{3}=0$. The second strategy, denoted RAW (rudder+aileron with), has the aileron deflected in the direction of the spin, or against the switching surface $\sigma_{1}=0$. The second strategy mimics the conventional method of spin recovery. The elevator is returned to the neutral position in both cases.

Figure 8 presents example flight test results for the RAA spin recovery control law along with the associated cell absorption probability plot. The open square in Fig. 8b marks the point at which the spin recovery control law is activated. The open triangle marks the end of the flight. The cell-to-cell map analysis reveals two unshaded 
absorbing cells. They are designated by the unshaded cells in Fig. 8a. The transient cells in Fig. 8a are shaded so that the darkest blue shades denote 2 steps of 0.35 second duration. As a result, the glider is expected to recover from the spin in less than one second. The flight test result confirms this result, but also shows that the path to recovery is not necessarily direct.

Flight test results for the RAW spin recovery control strategy are presented in Fig. 9. The plot of cell absorption times for this control law reveals two unshaded absorbing cells. These cells are located on the $\sigma_{3}=0$ switching surface with roll rates of approximately $3.5 \mathrm{rad} / \mathrm{s}(200 \mathrm{deg} / \mathrm{s})$. The transient cells shown in Fig. 9a are shaded such that the darkest shade represents 3 steps of 0.12 second duration. Consequently, the glider is expected to move from the spin critical point to the nearest absorbing cell in less than one-half second. Figure $9 \mathrm{~b}$ demonstrates that the glider response trajectory moves to the positive roll rate absorbing cell. The trajectory remains near the absorbing cell for a short period of time, before returning to the origin as the glider reaches the ground.

The effectiveness of the RAW spin recovery strategy is apparent in the response trajectories presented in Fig. 10. For comparison, time histories from the RAA flight test are shown in Fig. 10a and time histories from the RAW test are shown in Fig. 10b. The open circles in these plots represent the time at which the spin entry controls are applied. The open squares mark the time at which the spin recovery control is activated. Figure 10b shows that the pitch rate remains positive once the RAW recovery is applied. This characteristic was shown in earlier analysis to help the airplane move toward the $\sigma_{3}=0$ switching surface. The RAW control law forces the aircraft into a steady roll. Figure 9b demonstrates that a steady roll rate of about $200 \mathrm{deg} / \mathrm{s}$ is achieved in less than one-half second from the time that the RAW control is activated. The RAA control law does not appear to effectively reduce angle-of-attack. In fact, the glider angle-of-attack exceeds twenty degrees for nearly one second after the RAA control is activated. The angle-of-attack is fairly small when the RAW control law is activated, but one can see that this control law keeps the angle-of-attack close to zero throughout the remainder of the flight.
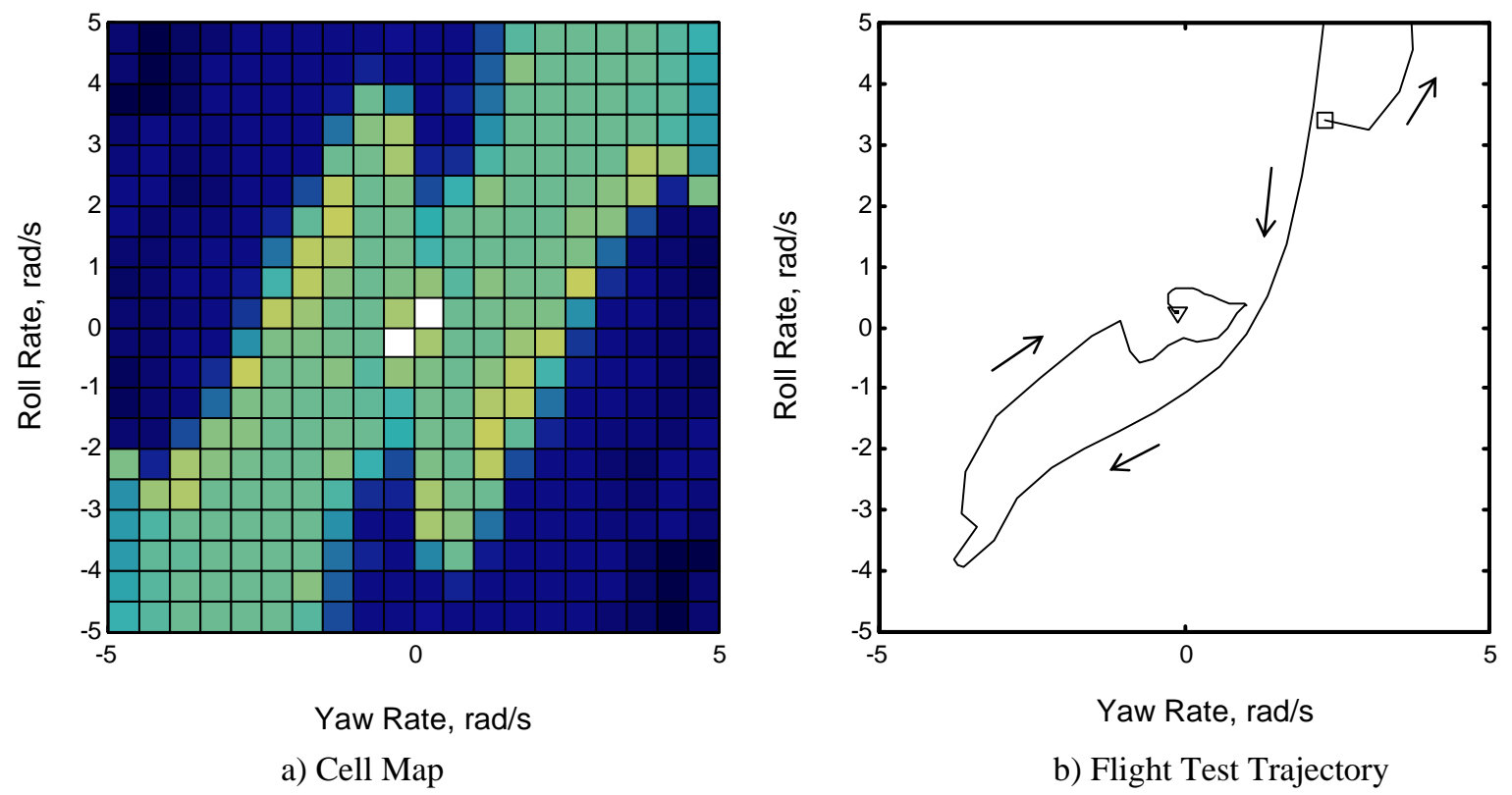

Figure 8. RAA Spin Recovery Cell Map and Flight Test Trajectory 


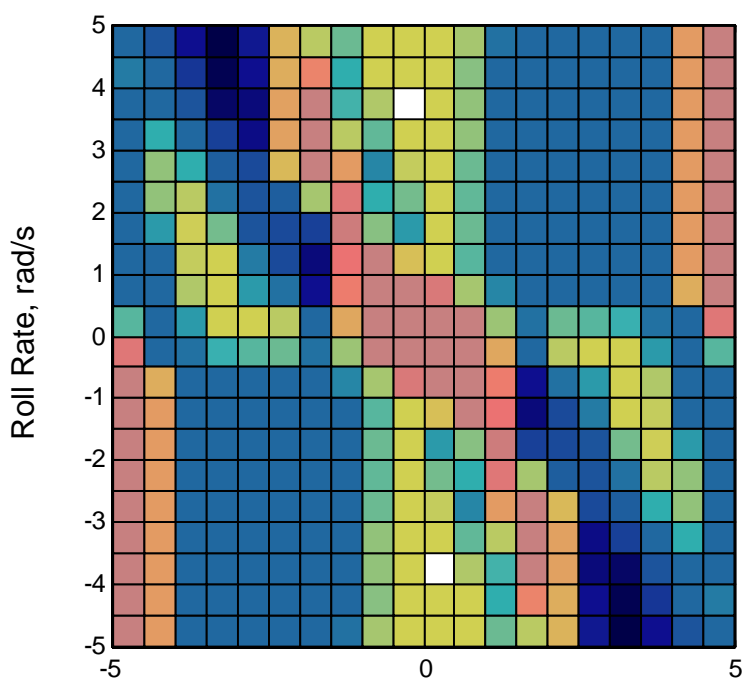

Yaw Rate, rad/s

a) Cell Map

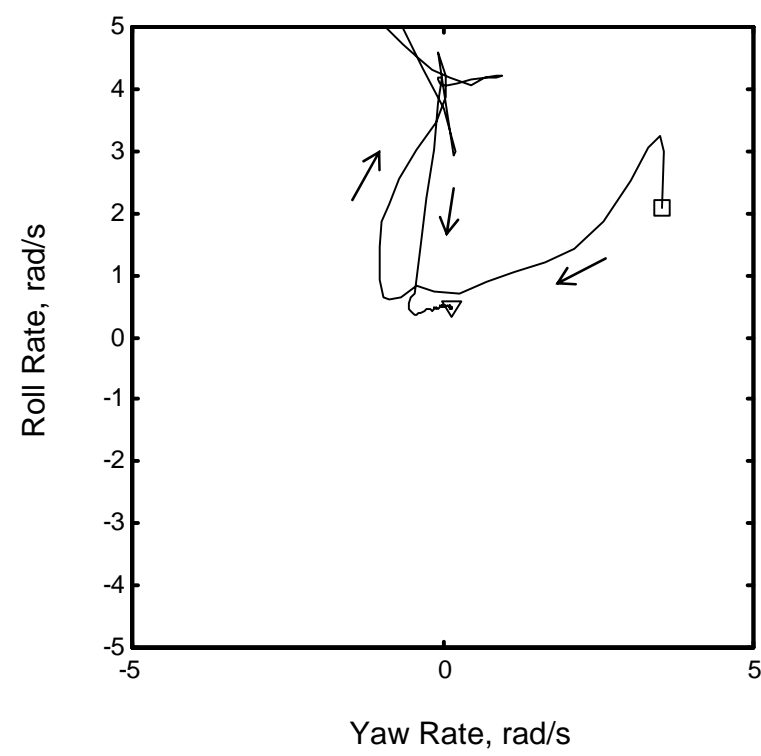

b) Flight Test Trajectory

Figure 9. RAW Spin Recovery Cell Map and Flight Test Trajectory
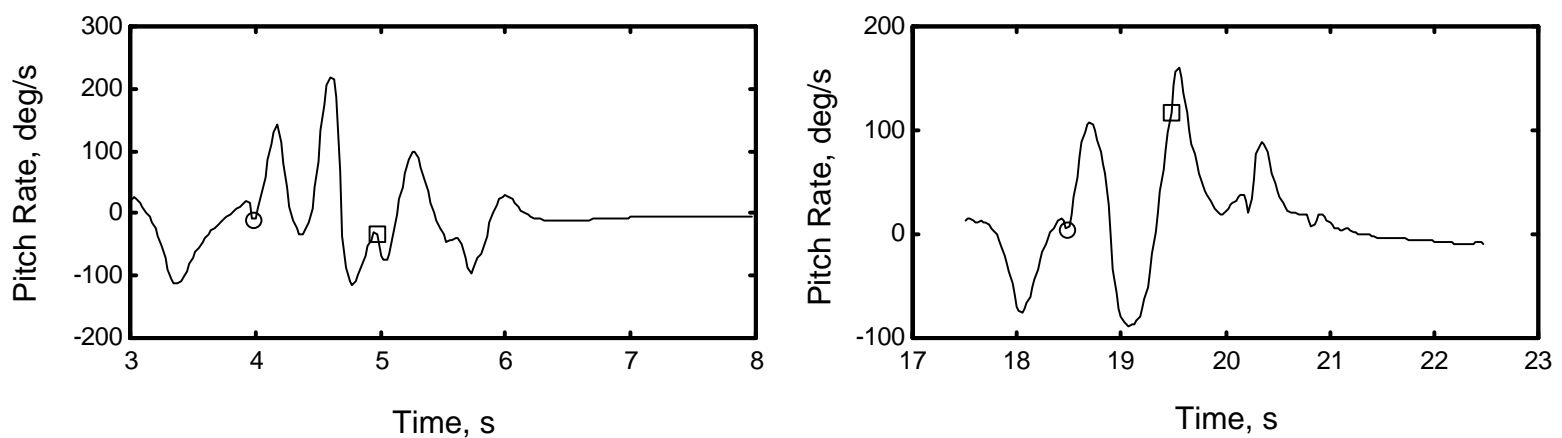

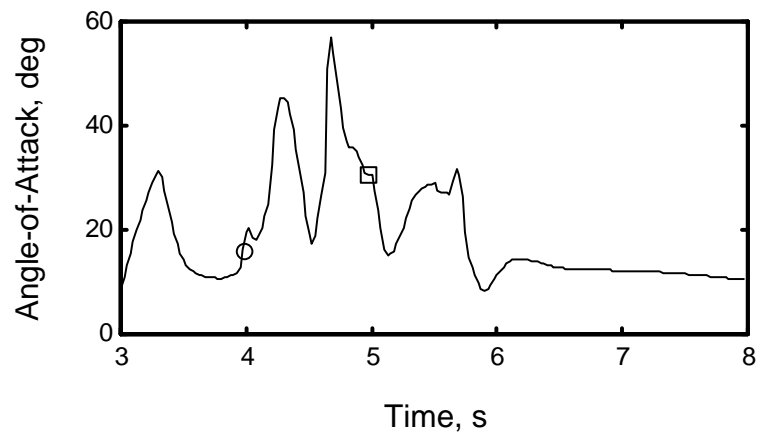

a) RAA Recovery

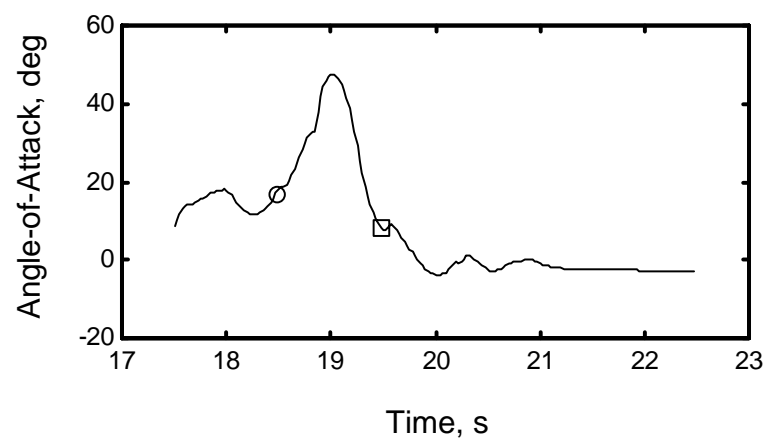

b) RAW Recovery

\section{Figure 10. Spin Recovery Trajectory Comparison}

It is interesting to compare the RAA and RAW recovery strategies from the perspective of a human pilot in a full-scale airplane. The RAA control law ultimately led to a complete recovery, but large excursions in the motion variables were encountered before the aircraft recovers. The RAW control law rapidly moves the aircraft from the spin critical point to a new critical point on the $\sigma_{3}=0$ switching surface. This strategy is probably preferred by pilots because yaw rate is eliminated first, which provides an immediate cue to the pilot that the recovery will be 
successful. An almost pure rolling motion at zero angle-of-attack defines the new critical point. Once the aircraft has reached this new critical point, the pilot completes the recovery using ailerons to stop the rolling motion. This second step represents the sliding mode of the VSC control scheme.

\section{Conclusion}

The value of an integrated approach to modeling, analysis, and control system design lies in the ease at which end-to-end flight dynamic studies can be conducted. The nonlinear lifting line modeling method yields a complete aerodynamic model yet requires only a small amount of basic geometric information. The subspace cell-to-cell mapping method gives a visual picture of the global stability properties near the chosen subspace. The dynamics of the state-space are readily manipulated using the theory and methods of variable structure control. The result is an integrated computational environment that can be used by a single investigator to rapidly explore flight dynamic issues related to large-amplitude maneuvers.

The end-to-end approach was demonstrated by investigating spin recovery control strategies for a small, handlaunched glider. This investigation showed that the recovery path could be manipulated by placing critical points at desired locations within the motion state-space. Variable structure control strategies were effectively used to shape the flow of trajectories to the critical point. The investigation confirmed that a conventional spin recovery technique, which requires the ailerons to be deflected with the spin, is a two-phase process that closely resembles a variable structure control law. The reaching mode phase defines a stable switching surface at zero yaw rate. During this phase, a simple relay control law drives the aircraft to a critical point that is almost pure rolling motion. The sliding mode phase control law would then eliminate the rolling motion, leading to a complete recovery. Flight test experiments verified the stability of the reaching mode phase.

\section{Acknowledgments}

The NASA Langley Research Center has supported this research under contract NAS1-02048. The authors would like to thank Dr. John B. Davidson for his careful review and content suggestions.

\section{References}

${ }^{1}$ Neihouse, A.I., "The Aileron as an Aid to Recovery from a Spin", NACA Technical Note No. 776, Sept. 1940.

${ }^{2}$ Piszkin, S.T. and Levinsky, E.S., "Nonlinear Lifting Line Theory for Predicting Stalling Instabilities on Wings of Moderate Aspect Ratio," N62269-75-C-0356, Naval Air Warfare Center, June 1975.

${ }^{3}$ Hsu, C.S., Cell-to-Cell Mapping: A Method of Global Analysis for Nonlinear Systems, Springer-Verlag, New York, NY, 1987.

${ }^{4}$ DeCarlo, R.A., Stanislaw, H.Z., and Matthews, G.P., "Variable Structure Control on Nonlinear Multivariable Systems: A Tutorial," Proceedings of the IEEE, Vol. 76, No. 3, March 1988, pp. 212-232.

${ }^{5}$ Anderson, M.R., "Modeling and Analysis of Large Amplitude Flight Maneuvers," NASA/CR-2004-212994, August 2004.

${ }^{6}$ Etkin, B., Dynamics of Atmospheric Flight, John Wiley \& Sons, Inc., New York, NY, 1972.

${ }^{7}$ Julier, S., Uhlmann, J., and Durrant-Whyte, H.F., "A New Method for the Nonlinear Transformation of Means and Covariances in Filters and Estimators," IEEE Transactions on Automatic Control, Vol. 45, No. 3, March 2000, pp. 477-482.

${ }^{8}$ Critzos, C.C., Heyson, H.H., and Boswinkle, W., "Aerodynamic Characteristics of NACA 0012 Airfoil Section at Angles of Attack from 0 to 180 Degrees," NACA TN 3361, Jan. 1955. 